Instructions for authors, subscriptions and further details:

http://qre.hipatiapress.com

\title{
La Relación de Investigación como una Relación entre Docentes. Una Indagación Narrativa
}

Ester Caparrós Martín ${ }^{1}$, Diego Martín-Alonso ${ }^{1}$ \& José Eduardo Sierra Nieto $^{1}$

1) Facultad de Ciencias de la Educación, Universidad de Málaga, España.

Date of publication: October $28^{\text {th }}, 2021$

Edition period: October 2021 - February 2022

To cite this article: Caparrós Martín, E., Martín-Alonso, D., \& Sierra Nieto, J. E. (2021). La Relación de Investigación como una Relación entre Docentes. Una Indagación Narrativa. Qualitative Research in Education, 10(3), 316-341. http://dx.doi.org/10.17583/qre.8479

To link this article: $h t t p: / / d x . d o i . o r g / 10.17583 /$ qre.8479

\section{PLEASE SCROLL DOWN FOR ARTICLE}

The terms and conditions of use are related to the Open Journal System and to Creative Commons Attribution License (CC-BY). 


\section{The Research Relationship as a Relationship between Teachers. A Narrative Inquiry}

Ester Caparrós Martín

Universidad de Málaga

José Eduardo Sierra Nieto

Universidad de Málaga
Diego Martín-Alonso

Universidad de Málaga

(Received: 01 June 2021; Accepted: 20 October 2021; Published: 28 October 2021)

\section{Abstract}

In recent decades, qualitative research has explored methodologies that go beyond the traditional expert-practical model, giving rise to participatory forms of research. A shift that, in the educational area, has taken shape in various formats, from action research to inquiry communities. In this context, we have developed a narrative inquiry that focuses on our research practices during a study on curriculum making in a primary school. In this paper, we pay attention to research relationships under the format of "research between teachers", which points to the recursion between research practices and teaching in teacher education. The research procedures have been: hermeneutical conversations, qualitative analysis of field texts and (re)writing. After the composition of a story-report on the research experience, we point out two issues in the discussion of results: (i) the creation of a relationship between teachers who investigate and (ii) listening as a research disposition. The article ends by delving into a phenomenological dimension of the research experience and its resonances for university teaching.

Keywords: qualitative research, narrative inquiry, hermeneutic phenomenology, research relationships, teacher education 


\section{La Relación de Investigación como una Relación entre Docentes. Una Indagación Narrativa}

Ester Caparrós Martín

Universidad de Málaga

José Eduardo Sierra Nieto

Universidad de Málaga
Diego Martín-Alonso

Universidad de Málaga

(Recibido: 01 de junio de 2021; Aceptado: 20 de octubre de 2021;

Publicado: 28 de octubre de 2021)

\section{Resumen}

En las últimas décadas, la investigación cualitativa se ha abierto a metodologías que escapan al tradicional modelo experto-práctico, dado lugar a formas participativas de investigación. Un giro que, en educación, se ha concretado en diversos formatos, desde la investigación acción a las comunidades de indagación. Bajo este escenario, hemos desarrollado una indagación narrativa que toma como foco nuestras prácticas de investigación durante el desarrollo de un estudio sobre creación curricular en una escuela de primaria. En dicha indagación, prestamos interés a las relaciones de investigación bajo el formato de investigar entre docentes, que apunta a la recursividad entre las prácticas de investigación y la docencia en la formación inicial del profesorado. Los procedimientos metodológicos utilizados han sido: las conversaciones hermenéuticas, el análisis cualitativo de los textos de campo y la (re)escritura. Tras la composición de un relato sobre la experiencia de la investigación, pasamos a señalar dos asuntos en la discusión de resultados: (i) la creación de una relación entre docentes que investigan y (ii) la escucha como disposición investigadora. El artículo finaliza ahondando en una dimensión fenomenológica de la experiencia de investigar y en sus resonancias para la docencia universitaria.

Palabras clave: investigación cualitativa, indagación narrativa, fenomenologíahermenéutica, relaciones de investigación, formación inicial del profesorado 
E

n el escenario actual de la investigación en ciencias sociales nos hemos familiarizado con metodologías que ofrecen un papel participante a personas o grupos que, con ello, dejan de ser objetos para posicionarse como sujetos. Así lo vemos en el hecho de disponer de varios handbooks que aglutinan, bajo el amplio paraguas de la investigación participativa, perspectivas en las que la relación sujeto-objeto desde una lógica positivista se ve desbordada (Denzin \& Lincoln, 2012; Noffke \& Somekh, 2009; Reason \& Bradbury, 2012). Reconocemos una estela de trabajos que arraigan en campos de conocimiento y realidades sociales plurales como son los procesos participativos (Villasante, 2006), la antropología colaborativa y el activismo (Arribas, 2015), el feminismo (Gatenby \& Humphries, 2000) o la educación (Darretxe et al., 2020; Elliott, 1993; Lieberman \& Miller, 2003). Un campo este último en el que identificamos dos ámbitos en los que la redefinición de las relaciones de investigación está muy presente: (1) la investigación inclusiva y los estudios sobre la discapacidad (Barton, 2005; García y Cotrina, 2020); y (2) la investigación de corte crítico-emancipador (Kincheloe, McLaren \& Steinberg, 2011) ${ }^{1}$. En este artículo nos centraremos en esta cuestión respecto del estudio de los saberes docentes (Contreras, 2010; Mercado, 1991; Tardiff, 2004).

Situándonos en este contexto, y como parte del desarrollo de dos proyectos de investigación en los que hemos participado (EDU2016-77576P; EDU2011-29732-C02-21) $)^{2}$, nos viene importando desarrollar formas creativas de investigación que, en línea con algunas de las tradiciones mencionadas, se preocupan por tejer relaciones investigativas que escapan al tradicional modelo experto-práctico (Blanco, 2010; Contreras, 1991). En este sentido, nos ha interesado avanzar en la formalización de un pensamiento y una práctica de la investigación que es sensible a la experiencia de exposición y de relación con la alteridad (Sierra et al., 2016). Una perspectiva que descansa en el reconocimiento del saber de las escuelas (Blanco, 2005); que busca afianzar aquellos vínculos que ayudan a conformar lo que Clandinin \& Connelly (1995) llaman comunidades de indagación; y qué, además, conecta con la importancia de que el conocimiento construido encuentre modos de filtrarse en la formación del profesorado (Blanco et al., 2015).

El trabajo en estos proyectos nos llevó a un primer desplazamiento en la creación de relaciones de investigación, dando forma a lo que nombramos 
estudios con docentes; una formalización que significaba esforzarnos en mantener con ellos y ellas una relación de pensamiento vivida, mientras atravesábamos la investigación como una experiencia -desde un punto de vista fenomenológico- (Blanco et al., 2016; Contreras \& Quiles, 2017; Contreras \& Pérez de Lara, 2010). Progresivamente, y como consecuencia de ir madurando en el seno del equipo las disposiciones y procedimientos de indagación, creamos otra modalidad que hemos nombrado como investigar entre docentes.

Para profundizar en esto hemos llevado a cabo una indagación narrativa que ha tomado como foco nuestras prácticas de investigación durante el desarrollo de un estudio sobre creación curricular (Clandinin \& Connelly, 1992) en una escuela de primaria $^{3}$. Así, nos planteamos una serie de preguntas de investigación que tenían que ver, sucintamente, con dos asuntos: (1) el desarrollo de la investigación como una experiencia que se vive y (2) la naturaleza de las relaciones de investigación, proponiendo la escucha como disposición metodología clave para la construcción de conocimiento pedagógico. A partir de ahí, el propósito de esta indagación es explorar aquellos modos de proceder en la investigación educativa que resultan autoconscientes y que se orientan a la construcción de comunidades de indagación desde el reconocimiento de las epistemologías docentes. Esto nos sitúa en la tradición de la indagación narrativa (Clandinin, 2013, 2017) y en la investigación fenomenológica (Contreras y Pérez de Lara, 2010; Van Manen, 2003).

\section{Marco Teórico-Metodológico}

El marco epistemológico que nos dibuja la investigación educativa invita a repensar la relación investigador-investigado, quedando abiertos a relaciones que se esfuerzan por reconocer al otro no como objeto de estudio, sino como sujeto coparticipe en la construcción de sentido (Bárcena, 2005; Belausteguigoitia y Mingo, 1999). Esta, que es una cuestión epistemológica, pasa a ser también un asunto ético, en tanto que nos demanda cuidar la relación con las personas con las que investigamos, como nos recuerda Parrilla (2010). En esta misma línea se expresan las dos tradiciones de investigación en las que nos apoyamos: (1) la indagación narrativa $\mathrm{y}$, dentro de esta, la noción de co-composición; y (2) la fenomenología hermeneútica. y, más concretamente, la escucha 
fenomenológica. Respecto al primer pilar, diremos que la indagación narrativa encuentra una abundante literatura desarrollada desde el Center for Research for Teacher Education and Development de la Universidad de Alberta (Clandinin, 2013; Clandinin \& Husu, 2017) y desde el grupo de investigación ESFERA de la Universidad de Barcelona (Contreras, 2016, 2017). En cuanto al segundo, encuentra su principal referente en la obra de Van Manen $(2003,2015)$ y en otros autores que han desarrollado su trabajo en una línea similar (Contreras, 2013; Larrosa \& Aparici, 2000; Quiles, 2016; Sierra \& Blanco, 2017).

Desde la indagación narrativa se entiende precisamente la narratividad como una cualidad ontológica de la experiencia humana, de tal modo que esta se (re)construye como un relato. Al "narrativizar la vida" (Bolívar, 2002) vamos construyendo historias que dan sentido a nuestra experiencia, a nuestro lugar en el mundo; de tal manera que la historia de vida de cada uno se va componiendo en el seno de un proceso dialógico, en las continuas relaciones con el entorno y el otro, y lo que nos vamos contando de ese vivir (Dewey, 1997).

En nuestro caso, ha sido con una maestra junto a quien hemos ido componiendo el sentido de la experiencia educativa en un estudio sobre creación curricular, entendiendo que lo real es fruto de esa construcción compartida que nace de la conversación. En consecuencia, el procedimiento de investigación que emerge desde este marco ontológico es el diálogo. Es por ello por lo que las conversaciones hermenéuticas se convierten en el procedimiento principal de la investigación (Sierra y Blanco, 2017; Van Manen, 2003); un dispositivo metodológico concebido como un encuentro en el que los investigadores no se limitan a recoger información para acabar "destapando una verdad", sino que se practica y cuida como un espacio de escucha mutua orientado a la reflexión y construcción compartida de sentido y de saber pedagógico (Kohler, 2008).

De acuerdo con lo anterior, desde la indagación narrativa se introduce la idea de co-composición con el propósito de reflejar que los textos de campo son creaciones elaboradas en las experiencias de relación entre investigadores y participantes; haciendo explícito también, que lo que pretenden narrar y mostrar son precisamente aspectos de esa experiencia de relación (Clandinin, 2013). Hay por tanto una dialéctica inscrita en la presencia del investigador o la investigadora como sujeto histórico que se pone en juego en una relación de alteridad. Un planteamiento en el que 
sobresale el principio de reconocimiento del otro o la otra como sujeto poseedor de un conocimiento valioso de carácter local (Cochran \& Lytle, 2003). A su vez, ese reconocimiento nos dispone a una escucha receptiva que, orientada por la noción de co-composición, debe ir desarrollándose como un diálogo en el que atribuir sentido a la experiencia -en este caso- de la enseñanza.

En definitiva, cuando hablamos de la escucha en el marco de la indagación narrativa nos referimos a un doble plano: la escucha de sí y la escucha del otro o la otra; de tal manera que la relación de investigación se teje como una obra a dos que deja ver el formato al que nos venimos refiriendo de investigar entre docentes. En este sentido, hacer de la investigación una experiencia de relación supone un desplazamiento que posibilita que quienes investigan se vivan en una experiencia que es conjunta a la vez que singular; pues hay una disposición a dejarse afectar por lo que la relación en sí misma ofrece y construye, permitiendo que se reconfiguren percepciones, saberes y actuaciones.

\section{Diseño y Desarrollo de la Investigación ${ }^{4}$}

Como decíamos en la introducción, el propósito de este texto es explorar modos de proceder en la investigación educativa que resulten autoconscientes y que se orienten a la construcción de comunidades de indagación desde el reconocimiento de las epistemologías docentes. Para ello, hemos puesto en marcha una indagación narrativa delimitando como foco nuestras prácticas de investigación durante el desarrollo de un estudio sobre creación curricular (Figura 1). 


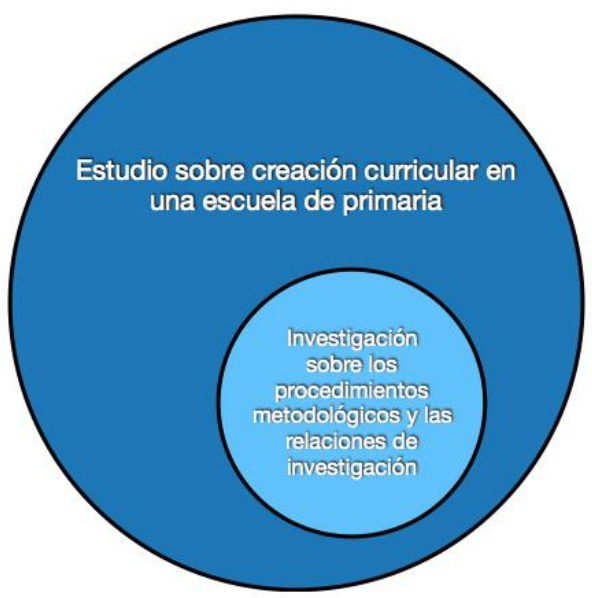

Figura 1. Investigación dentro de un estudio. Elaboración propia

Al tratarse de dos procesos metodológicos imbricados, creemos preciso recoger las distintas etapas atravesadas con el propósito de permitir al lector situarse en el diseño y el desarrollo de dichos procesos. Desde este marco, podemos hablar de 4 etapas (Figura 2):

- Una primera etapa coincide con el diseño y desarrollo del estudio del que es objeto nuestra investigación. Dicho estudio se proponía explorar la naturaleza del currículum vivido e investigar las condiciones que hacen posible una relación pedagógica que promueva la creación curricular (Martín-Alonso, 2019). Para ello, se procedió a acompañar a dos docentes, cada uno durante un curso escolar. En este texto nos centraremos en la relación de investigación que estableció el investigador de campo con una de las maestras, Clara, durante el curso 2017/18. Los procedimientos utilizados para ello fueron la observación de cerca y las conversaciones hermenéuticas (Van Manen, 2003); resultando este último clave para el foco aquí estudiado pues se trata de la creación de espacios de diálogo para la construcción de sentido sobre lo vivido en las escuelas. A partir de aquí es que se gestaron las preguntas de investigación. 
- Una segunda etapa corresponde con el análisis del estudio anteriormente citado, poniendo ya el foco en la experiencia de la relación de investigación. En esta etapa se llevó a cabo una lectura iterativa de los textos de campo: transcripciones de las entrevistas, el diario de campo (con anotaciones sobre las observaciones y las conversaciones) y el diario de investigación (que recogía aspectos relativos al desarrollo de la investigación y acerca de la propia relación de investigación). En esta etapa seguimos el procedimiento de análisis planteado por Marí et al. (2010) y Van Manen (2003). Para ello identificamos categorías temáticas, es decir, se agruparon unidades de significado de las transcripciones de las entrevistas y los diarios que giraban en torno a la relación de investigación y sus tensiones. En total se identificaron cuatro categorías temáticas: (i) el no-saber; (ii) la construcción de sentido; (iii) la escucha en diferido; (iv) reflejos en la docencia. La conceptualización de estas unidades de significado permitirá la construcción de un relato cuyo foco es la relación de investigación (Contreras, 2016).

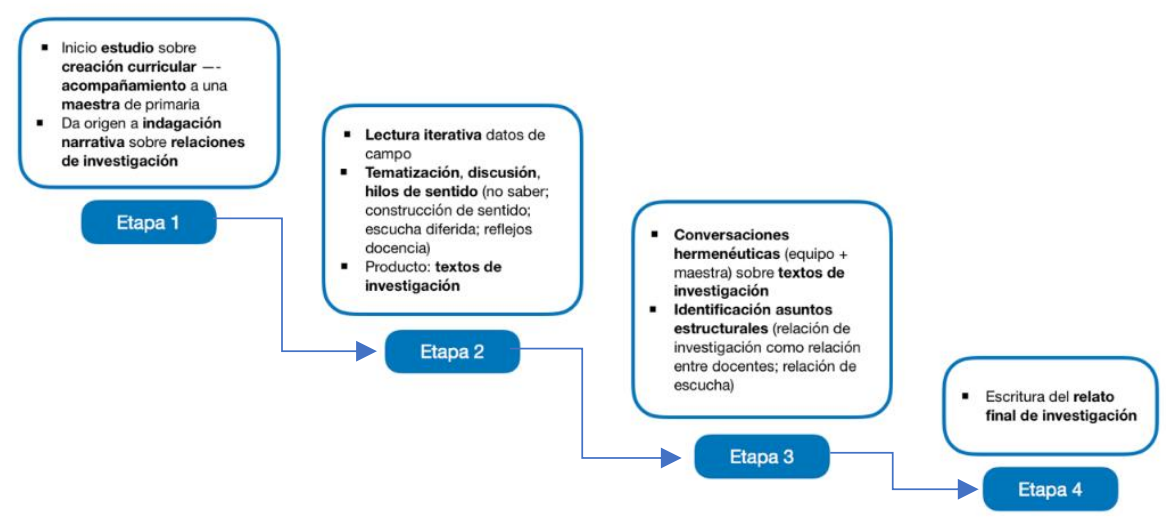

Figura 1. Etapas de la indagación narrativa. Elaboración propia

- La tercera etapa estuvo dedicada a la lectura y discusión del relato construido en la etapa anterior. En ella contamos con la participación de la maestra, siguiendo nuevamente la propuesta de Van Manen (2003) de las conversaciones hermenéuticas. Se repitió 
el procedimieno de análisis desarrollado en la etapa anterior: identificación de categorías temáticas del relato (Marí et al., 2010; Van Manen, 2003) y su conceptualización. El resultado de este trabajo fue la identificación de dos cuestiones estructurales: (i) la mirada a la relación de investigación como una relación entre docentes, en la que cada uno pone sus inquietudes en juego desde el lugar que ocupa (escuela o universidad); (ii) la necesidad de una disposición de escucha que posibilite una experiencia de investigación entendida como relación entre docentes.

- En la cuarta etapa, tras la identificación de estas dos cuestiones, se procede a la escritura del texto final, que presentamos en los siguientes apartados y que integra la reflexión sobre los dos asuntos estructurales señalados anteriormente. Como sugieren Clandinin (2013) y Sierra (2013), tanto en esta etapa de la indagación narrativa, como en la anterior, la escritura forma parte del procedimiento de análisis, en tanto que exige la reinterpretación del lugar, temporalidad y aspectos temáticos de los relatos.

\section{Relato: La Experiencia de una Relación de Investigación}

\section{EI No-Saber}

Lo primero que me encuentro al conocer a Clara es un vacío generativo y fértil. La relación comienza a crearse desde una suerte de desconocimiento pues, al preguntarle cómo construye los vínculos que apreciaba que mantenía con el alumnado, se repetía durante las primeras conversaciones una misma respuesta: "No sé".

Maestra: He sido una maestra querida por los alumnos. Yo me siento querida, no sé si lo he sido, que es diferente.

Investigador: ¿Y eso por qué crees que te quieren?

Maestra: Pues no sé. Primero los niños quieren, que no hay que hacerse muy orgulloso. Los niños quieren a cualquiera.

$[\ldots]$

Maestra: [Los niños me dicen] "seño, ¿pero es que tú nos quieres?" Investigador: ¿Cómo llega eso?

Maestra: Yo no lo sé. (E-12/01/2018) 
Al volver a pensar estos inicios de la investigación entiendo que, aunque pudiera ser frustrante, era una fase necesaria. Era frustrante porque resultaba fácil caer en la tentación de esperar encontrar las respuestas en la escuela, que la maestra me diese las claves de lo que andaba buscando. Pero si realmente íbamos a construir un sentido nuevo y plantearnos preguntas también nuevas, no podía esperar un discurso preparado. Debíamos partir del simbólico y repetido "no sé".

Tiene sentido, desde una mirada retrospectiva, la respuesta que ella daba: "Yo esas cosas no me las planteo así normalmente". Y es que se trata, en definitiva, de pensar juntos y construir sentido de lo vivido en relación con alguien con quien no se ha pensado antes o, al menos, no de esa manera. Se trata de "ordenar" el no-saber. Y el único modo para ello es "plantearse las cosas" de una manera que no se haya hecho antes, "que no se haga normalmente", en palabras de Clara.

No puedo saber con certeza qué preguntas se le abrían, ni hasta qué punto eran nuevas para ella, pero para mí la relación con la maestra me habría grandes incógnitas sobre el sentido de lo que ocurría en la escuela. Recuerdo, por ejemplo, el día en que una niña de $4^{\circ}$ lloraba sin consuelo mientras Clara seguía con su clase, como si no pasara nada; en la hora siguiente, fuimos a $2^{\circ} \mathrm{y}$, casualmente, otro alumno lloraba desconsolado. A este sí le atendió y dedicó, gran parte de la sesión, a cuidar lo que necesitaba para calmarse: ¿por qué atiende el llanto de unos y no de otros?, ¿es que un niño que llora no debe ser siempre atendido?, ¿por qué la niña que ha sido ignorada insiste días después en que "quiere" a Clara y que ella "la entiende"? La respuesta a todas las preguntas era la misma: no sé. Porque no es lo mismo la comunicación o el diálogo, que la relación. Y es que no se trata tanto de la aplicación de procedimientos de investigación como la conversación o la entrevista-, sino de un pensar en relación con alguien con quien nunca lo habíamos hecho lo que, a su vez, posibilita pensar sobre algo nuevo y desde un lugar diferente. Es gracias a esta relación que podemos adentrarnos en el descubrimiento del no-saber. Resulta revelador, en este sentido, el final de una de nuestras conversaciones, cuando salíamos del aula al final de la jornada escolar: 
Investigador: Qué interesante todo hoy.

Maestra: Es chulo. A mí me gusta hablar contigo porque me haces pensar y cosas que yo tengo, no tengo, no sé, me salen. (E23/02/2018)

\section{Construcción de Sentido}

Desde este modo de relación vamos pensando y van surgiendo ideas que posibilitan la comprensión de más aspectos de su docencia. Algunas quedan ahí y otras se siguen pensando ese mismo día y los siguientes. Por ejemplo, un día aparece la escucha como una cualidad que ella pone en juego en relación con sus estudiantes. Este será un hilo que continuará creciendo durante las siguientes conversaciones.

Maestra: Quizá es eso, intentar no dañar al otro. Intentar hacer las cosas con un fin positivo. No lo sé Diego, es difícil. Es muy difícil. Yo sé que tengo algo, yo me he dado cuenta con la edad. [...] Pues eso será algo, será magia, será física... No lo sé, lo llevo, o no lo llevo y quizá reboto lo que ellas tienen. Quizá es más la escucha.

Investigador: ¿Eso qué significa?

Maestra: A ver, ellos llegan a mí; pero igual que llegan a mí, llegan a ti. Y tú puedes hacer dos cosas: " $¡ a y$, sí!” y atenderlo, aunque sea leve; o le dices "luego". Cuando yo tres veces te digo "luego", ya tú has aprendido que a mí no vengas a decirme nada porque yo no te voy a hacer caso. Yo también creo que es un poco la escucha y la devuelta que se haga a esa escucha, aunque sea muy pequeña. (E$12 / 01 / 18)$

Surgido este tema, seguimos conversando y pensando en torno a él, en relación con lo que vamos viviendo juntos en el aula. Al ir pasando las semanas este termina convirtiéndose en un tema recurrente, al que vamos dando más sentido y significado, aunque no de forma estructurada. El tiempo va pasando, conozco a algunas de sus alumnas y converso con ellas. Estas niñas dicen reconocer a su maestra como una figura de autoridad (Blanco, 2015); alguien en quien confían, que las entiende y se preocupa por ellas. Por lo que, de vuelta a las conversaciones con Clara, este se convierte en otro hilo que orienta las conversaciones en torno a cómo cree que han llegado a construir esta relación de autoridad. Así, vuelve a 
aparecer la escucha y, con ella, lo que llamamos comprensión pedagógica. En un momento de la conversación reflexionaba Clara lo siguiente:

Es que si las maestras nos abrimos no podemos imaginar lo que podemos recibir, es que es increíble, es que siempre nos vamos a sorprender (E-14/03/2018).

Puedo decir que fui a la escuela preguntándome por la relación educativa y por las disposiciones que se ponen en juego, preguntas que quise explorar junto a Clara y en la propia escuela. Pero, después de este ejercicio hermenéutico terminé identificando determinados lugares que me señalaban el camino que debía continuar en la indagación; y entre ellos se encontraban los señalados aquí: la escucha y la comprensión.

\section{La Escucha en Diferido}

Después de mi paso por la escuela y las conversaciones con Clara, debía entrar en un proceso de transición a la escritura. El propósito radicaba en integrar narrativamente "una comprensión en retrospectiva de los hechos pasados, según la secuencia temporal continua, para llegar a un determinado fin" (Bolívar, 2002, p. 18). Este proceso, caracterizado por una suerte de escucha en diferido (Sierra \& Blanco, 2017), permanece en la relación pero sin presencia física, dando continuidad a la interpretación y la construcción de sentido. Y es que, en las conversaciones en directo, aunque envueltos en una tarea hermenéutica, nos encontramos presos "de las circunstancias y los apremios de la vida [de la escuela]" (Larrosa \& Aparici, 2000 , p. 40). De modo que no son interpretaciones realizadas desde la inmediatez de la conversación en directo, sino desde el sosiego y la soledad del estudio (Bárcena, 2020). Esto permite entrar en relación con lo vivido y conversado en distintos momentos, a la vez que se dialoga con la literatura pedagógica.

En este momento, aparecían textos que ya habían señalado a lugares que estaba estudiando, en particular al de la escucha y la comprensión. Tras su estudio, cuando volvía a las transcripciones podía encontrar signos nuevos, lugares de conexión con esos trabajos que me preceden. Así, entraba en una relación doble: por un lado, con la escucha en diferido de las conversaciones; por otro, con el pensamiento de otras autoras y otros 
autores. Esto me sucedió, entre otros, con los asuntos ya señalados de la escucha de la maestra. Por ejemplo, me resultó de gran valor el capítulo de Van Manen (1998) "La práctica de la enseñanza". En él, el autor desarrolla su idea de la "comprensión pedagógica", que expone de la siguiente manera:

La comprensión educativa se basa en la comprensión de cómo experimenta el niño el currículo, y en una evaluación de las fuerzas y debilidades en el aprendizaje del niño. El pedagogo tiene que saber cómo valorar las capacidades del niño en determinado momento, así como sus posibilidades. (ibíd., p. 106)

Precisamente esto era sobre lo que veníamos pensando en nuestras conversaciones. Desde las ideas y significantes que me ofrecía Van Manen podía nombrar lugares nuevos en la experiencia vivida con la maestra. Y eso me ha ido permitiendo pensar y escribir sobre la escucha, una tarea que se concretaba, por ejemplo, en uno de los artículos que componen la tesis y que tiene la escucha y la experiencia de Clara en la escuela como eje donde exponía que:

Escucha, aceptación y disponibilidad, constituyen un único gesto que, siguiendo las palabras de Laura [alumna], podríamos llamar como comprensión («era comprensiva, ella como que se ponía en tu piel», decía). Algo que hemos coincidido en llamar, junto con Van Manen (ibíd.), como comprensión pedagógica [...] Se trata de escuchar la historia del otro, aceptarla tal como la vive y (se) la cuenta para que, al entrar en relación, ésta pueda recrearse. Por tanto, es un gesto que se basa en la búsqueda de comprensión de la vida interior del otro, su experiencia vivida, el sentido que le da y, en último término, su currículum vivido. (Martín-Alonso et al., 2019, p. 120)

Por otro lado, en este proceso de "transición a la escritura" no busco descifrar lo que ahí hay sino, una vez más, construir sentido juntos, en relación. Y en tanto que vivo este proceso en primera persona, me dejo afectar, poniendo en juego mi historia. En consecuencia, todo este trabajo termina convirtiéndose en un modo personal de mirar a la educación y la relación educativa, construida, en este caso, desde una forma particular de 
entender la escucha; teniendo lugar lo que Biesta (2017) nombra como transcendencia.

\section{Reflejos en la Docencia}

Paralelamente a este proceso de investigación tenía docencia en la Facultad de Ciencias de la Educación. Y si la investigación realmente me ha transcendido, si ha supuesto cambios en la forma de entender la educación y la relación educativa, esto necesita abrirse a mi aula de formación: tanto en el modo en que me acerco a las estudiantes y las escucho, como en los propios saberes que pongo en circulación. De esta manera, podría decir que se produce un eco de aquello que vivía junto con Clara, una suerte de reverberación en mi docencia y con mis estudiantes. Se refleja esto, como decía, en los saberes y las preguntas que llevo a clase, preocupándome porque se formulen desde lugares en los que puedan empezar a mirar y pensar la práctica educativa como un oficio relacional.

Las reverberaciones de la investigación llegaban también a mi modo de entrar en relación con las estudiantes y situarme en el aula. De manera anecdótica, pero también simbólica, hasta ese curso me sentía incapaz de recordar el nombre de todas las estudiantes (por norma más de sesenta en cada grupo); sin embargo, desde ese año me surge la necesidad de conocer el nombre de todas -por difícil que sea-, como si con ello me acercarse más a ellas y sus historias. También resulta simbólico, por poner otro ejemplo, el cambio que realicé en mis clases, pasando de un uso predominante de la pizarra digital a la tradicional. Con la versión digital debía preparar las clases y los contenidos de una manera que se presentaban en un orden ya fijado por el material que había preparado (aunque hubiese alguna divagación, alguna pregunta nueva, siempre debíamos volver al hilo inicial para continuar lo ya estipulado). Cuando quise poner en juego todo lo que venía pensando en la relación de investigación necesité buscar un soporte que me permitiese escuchar a las estudiantes, lo que me proponían y adaptar la clase a sus inquietudes; por lo que decidí pasar a la pizarra tradicional, en la que puedo hacer y rehacer lo que llevo preparado en función de lo que va aconteciendo y escucho en clase.

En esta misma línea, mis estudiantes hacían mención en sus trabajos y tareas de evaluación final a estos dos lugares en los que trascendía mi relación de investigación con la maestra. Así pude observar que: (i) habían 
construido o comprendido la escucha como una cualidad del oficio docente; (ii) comentaban sentirse escuchadas y atendidas.

\section{Discusión}

Como hemos tratado de mostrar, la investigación entendida como cocomposición de saber pedagógico no se limita a una cuestión de fases y técnicas que aplicar, sino que tiene que ver más con una dotación de sentido en torno a aquello que se produce en el encuentro entre investigador y colaborador/a. Pero ¿qué disposiciones han posibilitado vivir la investigación como experiencia de creación conjunta? ¿Qué ha sido necesario poner en juego como investigador para que se produzca esta trascendencia (Biesta, 2017)?

En el relato aparece una escucha respetuosa que acepta al otro desde quien es; y una presencia solícita y consciente del investigador que desplaza su tarea hacia la creación de una relación de investigación con la maestra. Ambas son cualidades importantes que posibilitan a cada quien ser interpelado por la experiencia de investigar de un modo singular.

A continuación, se argumenta qué hay detrás de concebir la investigación como una relación entre docentes y qué ha significado la escucha pedagógica dentro de esa relación y para la práctica de la investigación.

\section{La Creación de una Relación entre Docentes que Investigan}

Dentro de procesos colaborativos de investigación es importante cuidar que estos no usurpen, no intimiden y no objetiven lo vivido (Sierra et al., 2016). Así, en el relato vemos como el investigador es alguien que pasa de la búsqueda de respuestas previstas, intencionales y prefijadas, a un dejarse afectar por lo que el encuentro con la maestra le supone. En este sentido, podemos decir que el proceso de investigación ha sido transitado como experiencia personal (Contreras \& Pérez de Lara, 2010), pues ha trascendido los límites del sí mismo; y no solo para el investigador, también para la maestra, aunque en modos distintos.

Lo anterior se deja ver bien en el relato cuando, paralelamente a su rol de investigador, este reconoce algunos movimientos que le han generado cambios en su práctica docente, ayudándole a resituarse en su oficio dentro 
de la formación del magisterio y en la relación con sus estudiantes; incluso, a ir configurando nuevas colocaciones en la práctica de investigación.

Para la investigación educativa, la naturaleza de la co-composición ofrece la posibilidad de una relación que, en cada uno de los implicados, es vivida y movilizada desde lo que Biesta (2017) denomina subjetividad profesional (p. 158). Como hemos ido viendo en el relato, el saber docente del investigador también está presente cuando va a la escuela y conversa con la maestra, pues quién se es como docente no se puede separar de la mirada con que se acerca al contexto de indagación y cómo se coloca en él. $\mathrm{Y}$ esto es importante rescatarlo pues docencia e investigación se imbrican en lo educativo, que es el terreno en el que nos movemos (Cochran-Smith \& Lytle, 2002). No obstante, para la maestra con la que investiga, el desplazamiento que ofrece el investigar con está en pasar de sentirse "sujeto investigado" a "sujeto creador de conocimiento" y tener la oportunidad de repensar su oficio y sus experiencias de otros modos.

El formato de investigación entre docentes invita entonces a los participantes a una comprensión y reflexión compartida de lo que se vive en las escuelas que resulta menos técnica y más ligada al sentido con el que piensan la educación en los diferentes contextos, escuela y universidad. Y es precisamente la particularidad del encuentro lo que posibilita formular nuevas preguntas y situarse en otros lugares desde los que mirar lo educativo.

El desplazamiento descansa entonces en pensar la educación en ese pasaje entre escuela y universidad (y viceversa); en eso que ocurre cuando la investigación universitaria se acerca a la escuela dejándose afectar por lo que el encuentro supone en tanto que relación. No se trata, pues, de investigar a la maestra y sus saberes docentes, sino investigar con ella sobre los saberes docentes; se trata de poner en el centro de la investigación educativa el conocimiento pedagógico de la práctica (Cochran-Smith \& Lytle, 2003).

La investigación, así entendida, se convierte en "un encuentro movido por propósitos pedagógicos que enraíza en la propia biografía y que conecta con la tradición y los bagajes culturales" (Sierra \& Blanco, 2017, p. 305). Y la relación de investigación -nuestro foco de atención particular en este artículo- se convierte en una experiencia que deshace la diferencia entre cognoscente y conocido, entre sujeto de estudio y objeto de estudio, para que se conjuguen y entren en diálogo. 


\section{La Escucha como Disposición Investigadora}

El docente reflexivo piensa sobre sí mismo, sobre su experiencia (Arévalo, 2010). Y aunque pueda acercarse a través de la lectura a otros autores y textos, al final volverá sobre sí. Por ello, para evitar una reflexión autorreferencial, se hace necesario salir de ese "sí mismo" e ir al encuentro y la conversación con otros docentes. Así, la investigación como una relación entre docentes permite precisamente esta salida del sí mismo, en tanto que se convierte en un encuentro con un profesional que se sitúa en otro lugar, permitiendo mirar a ese mismo lugar (el foco de estudio) pero desde un sitio diferente.

Según lo analizado, se hace necesario señalar una disposición que consideramos hace posible la investigación como experiencia compartida: la escucha. Una escucha que requiere la aceptación del otro, lo que trae y lo que nos invita a pensar. Diversos autores (Larrosa \& Aparici, 2000; Sierra \& Blanco, 2017) la orientan no a un "conocer" lo que el otro dice para recoger la información en la entrevista, sino como un dejarse decir o afectar. Es por eso por lo que nos ha parecido más preciso hablar de conversaciones hermenéuticas y no de simples entrevistas.

Esta clase de relación no es posible si uno se siente cosificado en tanto que objeto de estudio, o si se percibe dentro de una jerarquía de posiciones en las que su labor se pueda ver juzgada o puesta en entredicho. Al contrario, cuando se vive como sujeto de la investigación, en tanto que parte activa del pensar sobre su práctica, es que puede sentirse reconocido, abriéndose a verdaderos procesos reflexivos. Así enfocada, la escucha consiste en una disposición de acogida del otro, de sus inquietudes y de sus modos de mirar; disposición que nos invita a mirar, a pensar y cuestionar cómo nos moviliza lo que nos ofrece.

Vemos este movimiento de escucha en la expectativa inicial del investigador ante los continuos "no sé" de la maestra, donde sentía dificultades para encontrar respuestas rápidas y fáciles que le dijeran, de algún modo, qué significaba ese no-saber con el que ella le abría a lo educativo; obligándole a estar expectante a lo que sucediese y le sucediese. A partir de ahí, la relación gestada entre ambos y el ejercicio de escucha cultivado acabó desplazando la mirada y situó al investigador en una actitud receptiva respecto de lo que ella le ofrecía y daba a pensar. Por ejemplo, 
para comprender el sentido con el que Clara iba mostrándose en relación con su alumnado, el investigador trataba de hacerse preguntas que creía estaban relacionadas, en un proceso recursivo de ir a la literatura pedagógica y a la escuela.

La relación de diálogo que continuaba le abría, pues, a nuevos sentidos que debían ser pensados en la conversación y de vuelta a las preguntas de investigación, esta vez desde las categorías temáticas que iban apareciendo: ¿por qué las alumnas decían confiar en Clara?, ¿por qué la reconocían cómo figura de autoridad?, ¿era escucha?, ¿era empatía? Y así la investigación iba tomando forma, orientada por una actitud fenomenológica de escucha que daba espacio a los nuevos hilos que aparecían, sustanciando también la experiencia vivida en el aula.

La escucha en la relación de investigación supone, entonces, aceptar lo emergente de la investigación y lo contingente de la experiencia. Debe haber un plan de investigación inicial que guíe el proceso, pero debemos estar alerta para acoger lo inesperado en él. Se trata de crear vacío, de dar lugar a lo que traiga el otro, sin buscar ni pretender nada más que el encuentro. Un vacío que, dirán Larrosa \& Aparici (2000) “[...] es apertura y que por eso se abre hacia adentro, un vacío que hay que hacer en uno mismo, interrumpiendo el siempre demasiado lleno del saber y deteniendo el siempre demasiado ansioso del buscar" (p. 45). No obstante, no se trata de "dejar pasar" todo lo que aparezca, sino de generar un diálogo reflexivo y riguroso con lo que va aconteciendo en las escuelas y el propio avance de la investigación; haciendo un trabajo intelectual riguroso de pensar en directo y en diferido. Esta suerte de aceptación reflexiva es imprescindible para delimitar la investigación, ya que "el riesgo de apertura ad infinitum no deja de pender como una espada de Damocles sobre el proceso de investigación" (Cárcamo, 2014, p. 7).

\section{Conclusiones}

Al buscar problematizar las relaciones sujeto-objeto desde el interés por construir investigaciones de corte participativo a través de las que reconocer y recrear los saberes docentes, nos sumergimos en una indagación narrativa que tomaba como foco de estudio nuestras prácticas de investigación dentro de un estudio más amplio sobre creación curricular. 
Esta búsqueda fue cristalizando en la formalización de un tipo de relaciones de investigación que nombramos como investigar entre docentes.

Este proceso de pensar la práctica de investigación nos aproxima a un modo de indagación donde la presencia del investigador o la investigadora, así como la atención y el cuidado de las relaciones con la alteridad, significa disponerse a una comprensión sensible de aquello que se investiga y junto a quien se investiga; atendiendo, a su vez, a lo que dicha experiencia de relación supone para el propio investigador.

El proceso de indagación seguido nos ha servido para esclarecer cuestiones estructurales de esta forma de practicar la investigación educativa (Blanco, 2010) que tienen que ver con los dos asuntos centrales que nos movieron desde el principio: (1) el desarrollo de la investigación como una experiencia que se vive y (2) la naturaleza de la relación de investigación movida por la escucha como disposición metodológica.

Hemos planteado a lo largo del artículo que la investigación educativa, inscrita en la corriente interpretativa, reconoce la presencia del investigador o de la investigadora como un sujeto que se expone a relaciones de alteridad. Algo a lo que hacen mención Contreras \& Pérez de Lara (2010) cuando sostienen que

[...] la investigación pedagógica es siempre una forma de autoinvestigación: la realizamos desde nuestra visión de lo educativo, poniéndola en juego (poniéndonos en juego nosotros, con nuestras ideas, aspiraciones, formas de entender y hacer), y en el proceso investigador, en la aspiración a abrirnos a nuevas experiencias y comprensiones de lo que supone la educación, en el deseo de entender algo que no entendíamos (y por eso investigamos: para entender o ver de nueva manera algo que no entendíamos), nos exponemos. (p. 48)

Este posicionamiento coincide en gran medida con las palabras de Van Manen (2003) a propósito de que la investigación educativa "contribuye a la formación del propio carácter reflexivo y a la capacidad de uno mismo para actuar por y para los demás, niños o adultos, con tacto o discreción" (p. 25). En este sentido, continúa, "la investigación en ciencias humanas es en sí mismo un tipo de Bildung o paideia; es decir, el currículo del ser y el devenir" (ibíd.). 
Dicha concepción de la presencia del investigador o la investigadora como sujeto en formación se deja pensar en dos sentidos. En primer lugar, en cuanto a que la naturaleza de la relación de investigación desborda la relación experto-práctico para inaugurar una relación entre profesionales de la educación. Una relación motivada por un principio de reconocimiento de las epistemologías docentes y orientada por una disposición receptiva concretada en la idea de escucha. En nuestra indagación, esa relación entre docentes ha sido enfocada y vivida como una relación de construcción compartida de sentido, a través de la noción de co-composición; y ha servido para avanzar en la conformación de un trabajo colaborativo que se deja pensar bajo la idea de comunidades de indagación. En segundo lugar, la concepción de una investigación como experiencia de formación alude a la idea de trascendencia y a cómo los procesos de aprendizaje y transformación que se desarrollan en la investigación encuentran resonancias en la formación universitaria; es decir, investigamos sobre aquello mismo que enseñamos (Blanco et al., 2015). En este sentido, más que investigadores podríamos afirmarnos como docentes que investigan, cuya experiencia de investigar articula su enseñanza. De tal manera que poco sentido tiene la elaboración de exhaustivos informes de investigación si a la postre no encarnamos los saberes que allí se recogen o si la investigación no tiene finalmente como faro la mejora de la formación del profesorado.

Podemos decir, para finalizar, que la investigación educativa difícilmente puede sustantivarse como un programa de investigación, sino que merece ser considerada como una praxis en la que nos hacemos a nosotros mismo como educadores (Caparrós \& Sierra, 2012). Al tiempo que se planifican y ponen en marcha procedimientos que velan por el rigor y la credibilidad de nuestras prácticas, los modos de implicación personal desde el cultivo de una justa distancia- nos involucran de la manera en que lo expresábamos anteriormente con Van Manen. Y esto es así porque el conocimiento pedagógico es invariablemente el saber de alguien que están implicado en prácticas que se interrogan por el sentido de una experiencia para generar un conocimiento pedagógico que siempre es personal (Contreras 2010). Cobrando así sentido la recursividad de la investigación $\mathrm{y}$, de forma más concreta, la modalidad de las relaciones de investigación como relaciones entre docentes. 


\section{Notas}

${ }^{1}$ Complementariamente podemos identificar un tercer ámbito que conecta este asunto con perspectivas decoloniales (Rivas et al, 2020).

${ }^{2}$ Relaciones educativas y creación del currículum: entre la experiencia escolar y la formación inicial del profesorado. Indagaciones narrativas. Proyecto perteneciente al Plan Nacional de I+D+i del Ministerio de Economía y Competitividad (EDU2016-77576-P); El saber profesional en docentes de educación primaria y sus implicaciones en la formación inicial del profesorado: estudios de casos (EDU2011-29732-C02-21), Ministerio de Economía y Competitividad.

${ }^{3}$ Con el propósito de facilitar la lectura, a lo largo del artículo utilizaremos la palabra estudio para referirnos a la investigación sobre la creación curricular y la palabra investigación para referirnos al proceso de indagación sobre las disposiciones y los procedimientos metodológicos, núcleo de este texto.

${ }^{4}$ Conviene precisar que funcionar como equipo de investigación no ha significado homologar la experiencia vivida por el investigador que ha realizado el trabajo de campo; ya que la experiencia en sí del trabajo de campo es diferente de la de hacer pensamiento compartido en torno a ella. Atendiendo a esto, hemos cuidado que la escritura del relato de investigación respete la primera persona, en tanto que es narrada por el investigador implicado; aunque, como decimos, detrás de ésta haya una composición colectiva de ideas.

${ }^{5} \mathrm{La}$ codificación de las notas de campo se ha realizado siguiendo el siguiente esquema: (E de entrevista - fecha). El nombre de la maestra (Clara) es un pseudónimo para garantizar la confidencialidad y el anonimato.

\section{References}

Arévalo, A. (2010). La experiencia de sí como investigadora. En J. Contreras \& N. Pérez de Lara (Comps.). Investigar la experiencia educativa (pp. 188-198). Morata.

Arribas, A. (2015). Antropología colaborativa y movimientos sociales: construyendo ensamblajes virtuosos entre sujetos en proceso. Ankulegi 19, 59-73. https://aldizkaria.ankulegi.org/index.php/ankulegi/article/view/77

Bárcena, F. (2005). La experiencia reflexiva en educación. Paidós. Bárcena, F. (2020). El profesor en el estudio. Márgenes, Revista de Educación de la Universidad de Málaga, 1(2), 193199. https://doi.org/10.24310/mgnmar.v1i2.9612

Barton, L. (2005) Emancipatory research and disabled people: some observation and questions. Educational Review, 57(3), 317-327. 
Belausteguigoitia. M., \& Mingo, A. (eds.) (1999). Géneros prófuigos. Feminismo y educación. Paidós.

Biesta, G. (2017). El bello riesgo de educar. SM.

Blanco, N. (2005). Innovar más allá de las reformas. REICE, Revista Electrónica Iberoamericana sobre Calidad, Eficacia y Cambio en Educación, 3 (1). https://www.redalyc.org/pdf/551/55130137.pdf Blanco, N. (2010). La investigación en el ámbito del curriculum y como método para su desarrollo. En J. Gimeno-Sacristán, J. (Coord.). Saberes e incertidumbres sobre el curriculum (pp. 569-587). Morata. Blanco, N. (2015). Reconocer autoridad femenina en la educación: Los saberes de las maestras. Revista Qurriculum, 28, 11-31. http://qurriculum.webs.ull.es/wp-content/uploads/2015/04/01Nieves-Blanco.pdf

Blanco, N., Molina, M. D., \& Arbiol, C. (2016). Explorar las tensiones para transitar el abismo: Buscando el sentido del oficio docente. En J. Contreras, Tensiones fructíferas: Explorando el saber pedagógico en la formación del profesorado: Una mirada desde la experiencia (pp. 223-253). Octaedro.

Bolívar, A. (2002). “De nobis ipsis silemus?”: Epistemología de la investigación biográfico-narrativa en educación. Revista Electrónica de Investigación Educativa, 4(1), 1-26. Recuperado de: http://www.scielo.org.mx/scielo.php?script=sci_arttext\&pid=S1607$40412002000100003 \& \operatorname{lng}=$ es\&tlng=es

Caparrós, E., \& Sierra, J. E. (2012). Al hilo de lo vivido: la pedagogía como brújula para la investigación educativa. En I. Rivas, F. Hernández, J. $\mathrm{M}^{\mathrm{a}}$ Sancho \& C. Núñez (Coords.). Historias de vida en educación: sujeto, diálogo, experiencia (pp. 61-66). Dipòsit Digital UB REUNI+D. http://hdl.handle.net/2445/15323

Cárcamo, H. (2014). Acerca de la producción de conocimiento en ciencias sociales y los entretelones del enfoque etnográfico. En H. Cárcamo (Ed.), Making of... Construcciones etnográficas de la educación. Traficantes de sueños.

Clandinin, D. J. (2013). Engaging in narrative inquiry. Left Coast Press. Clandinin, D. J., \& Connelly, F. M. (1995). Relatos de experiencia e investigación narrativa. En J. Larrosa \& otros, Déjame que te cuente. Ensayos sobre narrativa y educación (pp. 11-59). Laertes. 
Clandinin, D. J., \& Connelly, F. M. (1992). Teacher as curriculum maker. En P. W. Jackson (Ed.), Handbook of research on curriculum: A project of the American Educational Research Association (pp. 363401). MacMillan.

Clandinin, D. J., \& Husu, J. (Eds.). (2017). The Sage handbook of research on teacher education. SAGE.

Cochran-Smith, M., \& Lytle, S. (2002). Dentro / Fuera. Enseñantes que investigan. Akal.

Cochran-Smith, M., \& Lytle, L. (2003). Más allá de la certidumbre: Adoptar una actitud indagadora. En A. Lieberman \& L. Miller (Eds.), La indagación como base de la formación del profesorado y la mejora de la educación (pp. 65-80). Octaedro.

Contreras, J. (1991). El sentido educativo de la investigación. Cuadernos de Pedagogía, 196, 61-67.

Contreras, J. (2010). Ser y saber en la formación didáctica del profesorado:

Una visión personal. Revista interuniversitaria de formación del profesorado, 68, 61-82.

https://www.redalyc.org/pdf/274/27419198004.pdf

Contreras, J. (2013). El saber de la experiencia en la formación inicial del profesorado. Revista Interuniversitaria de Formación del Profesorado, 78(27.3), 125-136.

https://dialnet.unirioja.es/servlet/articulo?codigo $=4688508$

Contreras, J. (2016). Tener historias que contar: Profundizar narrativamente la educación. Roteiro, 4l(1), 15-40.

https://doi.org/10.18593/r.v41i1.9259

Contreras, J. (Ed.). (2017). Enseñar tejiendo relaciones: Una aproximación narrativa a los docentes y a sus clases de Educación Infantil y Primaria. Morata.

Contreras, J., \& Pérez de Lara, N. (2010). Investigar la experiencia educativa. Morata.

Darretxe, L., Gezuraga, M., \& Berasategui, N. (2020). La necesidad de avanzar hacia la investigación inclusiva. Márgenes, Revista de Educación de la Universidad de Málaga, 1(1), 104-114. http://dx.doi.org/10.24310/mgnmar.v1i1.7139

Denzin, N. \& Lincoln, Y. (2012). Paradigmas y perspectivas en disputa. Manual de Investigación Cualitativa - Vol. II. Gedisa.

Dewey, J. (1997). Experience and education. Touchstone. 
Elliott, J. (1993). El cambio educativo desde la investigación - acción. Morata.

García, M. y Cotrina, M. (2020). “¿A qué llamamos investigación inclusiva hoy?”. En E. Caparrós, M. Gallardo, N. Alcaraz y A. L. Rizzo (Coords.) Educación inclusiva: un desafío y una oportunidad para la innovación educativa en la formación inicial del profesorado (pp. 69-81). Octaedro.

Gatenby, B., \& Humphries, M. (2000) Feminist participatory action research: Methodological and ethical issues. Women's Studies International Forum, 23(1), 89-105. https://doi.org/10.1016/S02775395(99)00095-3

Kincheloe, J., McLaren, P., \& Steinberg, S. (2011). Critical pedagogy and qualitative research. Moving to the bricolage. En N. Denzin \& I. Lincoln (eds.). The SAGE Handbook of Qualitative Reseach (163177). SAGE.

Larrosa, J., \& Aparici, B. (2000). Aprender de oído el aula, el claro y la voz en María Zambrano. Revista Educación y Pedagogía, 12(26-27), 3746.

https://revistas.udea.edu.co/index.php/revistaeyp/article/view/325112 Lieberman, A., Miller, L., \& Cercadillo, P. (2003). La indagación: como base de la formación del profesorado y la mejora de la educación. Octaedro.

López, A., Porres, A., Durán, N., Moltó, Ó. J., Díaz, G., Simó, N., \& Valls, M. (2013). Tránsitos epistemológicos y metodológicos en una investigación sobre el abandono escolar en la Educación Secundaria a partir del encuentro con los jóvenes. Revista de Educación, 360, 624-644. https://www.educacionyfp.gob.es/revista-deeducacion/numeros-revista-educacion/numerosanteriores/2013/re360/re360-28.html

Kohler, C. (2008). Narrative methods for the human science. SAGE. Marí, R.; Bo, R. M. y Climent, C. I. (2010). Propuesta de análisis fenomenológico de los datos obtenidos en la entrevista. Revista de Ciències de l'Educació, 113-133.

Martín-Alonso, D. (2019). El tejido curricular. Indagación narrativa sobre la relación educativa y el proceso de creación curricular (Tesis doctoral). Universidad de Málaga. https://hdl.handle.net/10630/19311 
Martín-Alonso, D., Blanco, N., \& Sierra, J. E. (2019). Comprensión pedagógica y construcción de la relación educativa. Una indagación narrativa. Teoría de la Educación. Revista Interuniversitaria, 31(1), 103-122. http://dx.doi.org/10.14201/teri.19442

Mercado. R. (2010). Los saberes docentes en el trabajo cotidiano de los maestros. Infancia y Aprendizaje, 55, 59-72. https://doi.org/10.1080/02103702.1991.10822305

Noffke, S., \& Somekh, B. (2009). The SAGE handbook of educational action research. SAGE.

Parrilla, Á. (2010). Ética para una investigación inclusiva. Revista de Educación inclusiva, 3(1), 165-174.

Quiles, E. (2016). Cuidar la relación: El sentido de la experiencia educativa. Una investigación narrativa. (Tesis doctoral). Universitat de Barcelona.

http://diposit.ub.edu/dspace/bitstream/2445/102098/1/EQF_TESIS.p df

Reason, P., \& Bardbury, H. (2012). The SAGE Handbook of Action Research Participative Inquiry and Practice. (2 ${ }^{\mathrm{a}}$ Ed.). Sage.

Rivas Flores, J. I., Márquez García, M. J., Leite, A. E., \& Cortés González, P. (2020). Narrativa y educación con perspectiva decolonial. Márgenes Revista De Educación De La Universidad De Málaga, 1(3), 46-62. https://doi.org/10.24310/mgnmar.v1i3.9495

Sierra, J. E. (2013). La Secundaria vivida. Estudio narrativo sobre experiencias masculinas de fracaso escolar (Tesis doctoral). Universidad de Málaga. https://riuma.uma.es/xmlui/handle/10630/6739

Sierra, J. E., \& Blanco, N. (2017). El aprendizaje de la escucha en la investigación educativa. Qualitative Research in Education, 6(3), 303-326. http://dx.doi.org/10.17583/qre.2017.2783

Sierra, J. E., Caparrós, E., \& Díaz, N. (2016). Nuevas miradas en investigación educativa: Indagar pedagógicamente la experiencia. International Journal of Educational Research and Innovation, 5, 184-194.

https://www.upo.es/revistas/index.php/IJERI/article/view/1624

Tardiff, M. (2004). Los saberes del docente y su desarrollo profesional. Narcea. 
Van Manen, M. (1998). El tacto en la enseñanza: El significado de la sensibilidad pedagógica. Paidós.

Van Manen, M. (2003). Investigación educativa y experiencia vivida: Ciencia humana para una pedagogía de la acción y la sensibilidad. Idea Books.

Van Manen, M. (2015). Pedagogical tact: Knowing what to do when you don't know what to do. Left Coast Press.

Villasante, T. (2006). Desbordes creativos: estilos y estrategias para la transformación social. La Catarata.

Ester Caparrós Martín is Assistant Professor Doctor in the Department of Didactics and School Organization at Faculty of Education Sciences at University of Málaga, Spain. ORCID: 0000-0003-1700-3577

Diego Martín-Alonso is Interim Substitute Professor in the Department of Didactics and School Organization at Faculty of Education Sciences at University of Málaga, Spain. ORCID: 0000-0001-7367-7862

José Eduardo Sierra Nieto is Assistant Professor Doctor in the Department of Theory and History and Methods of Research and Diagnosis in Education at Faculty of Education Sciences at University of Málaga, Spain. ORCID: 0000-0002-9925-1656

Contact Address: Ester Caparrós Martín, University of Málaga, Faculty of Education Sciences, Blvr. Louis Pasteur, 25, 29010 Málaga, Spain. Email: ester.caparros@uma.es 